\title{
WhatsApp Messenger use in oncology: a narrative review on pros and contras of a flexible and practical, non-specific communication tool
}

\author{
Vittorio Gebbia ${ }^{1,2}$, Dario Piazza ${ }^{3}$, Maria Rosaria Valerio ${ }^{4}$ and Alberto Firenze ${ }^{2,5}$ \\ ${ }_{1}^{1}$ Medical Oncology Unit, La Maddalena Clinic for Cancer, Palermo, Italy \\ 'Department of Internal Medicine 'Promise', University of Palermo, Palermo, Italy \\ ${ }^{3}$ GSTU Foundation for Cancer Research, Palermo, Italy \\ ${ }^{4}$ Medical Oncology Unit, Policlinic 'P. Giaccone', University of Palermo, Palermo, Italy \\ ${ }^{5}$ Risk Management Unit, Policlinic 'P. Giaccone', University of Palermo, Palermo, Italy
}

\section{Abstract}

The spread of instant messenger systems provides an excellent opportunity and a helpful tool to healthcare professionals. WhatsApp instant messenger use is widely prevalent among health professionals, cancer patients, caregivers and the general population. It is a quick and easy communication tool that may also be used on personal computers and business purposes. WhatsApp instant messenger and other similar tools may be a very useful complement for e-medicine. Instant messaging systems may be helpful, especially in rural areas, in medium- or low-income countries, or to avoid unnecessary travels, improve knowledge and awareness of cancer, monitor home care and support the delivery of home care. The unregulated use of WhatsApp instant messenger requires sound and shared guidelines to assure impeccable professional service. Although a significant number of papers have investigated the roles of social networks in connecting patients to health professionals, there is still a lack of information and scientific data about their uses, benefits and limitations in connecting health providers only for professional communication. The role of instant messenger systems in cancer practice and research needs to be clarified. In this paper, we report a focus on available data, pros and contras of the unregulated use of WhatsApp instant messaging, in the context of e-medicine, as an interprofessional and doctor/patient communication tool in oncology.

Keywords: WhatsApp, instant messenger systems, social media, telemedicine, cancer care, health professional's interaction, cancer research

\section{Introduction}

In the last decade, web-based technology has conquered an ever more significant space in oncology healthcare organisations and scientific research worldwide [1]. The COVID-19

Correspondence to: Vittorio Gebbia Email: vittorio.gebbia@gmail.com

ecancer 2021, 15:1334

https://doi.org/10.3332/ecancer.2021.1334

Published: $13 / 12 / 2021$

Received: 11/05/2021

Publication costs for this article were supported by ecancer (UK Charity number 1176307).

Copyright: (c) the authors; licensee ecancermedicalscience. This is an Open Access article distributed under the terms of the Creative Commons Attribution License (http:// creativecommons.org/licenses/by/4.0), which permits unrestricted use, distribution, and reproduction in any medium, provided the original work is properly cited. 
pandemic has caused a profound alteration or even disrupted the usual ways of communication between patients, caregivers, health professionals and oncological institutions [2]. In this tragic scenario, telemedicine and web-based tools have undergone an even more notable expansion than usual. These tools can minimise face-to-face contacts, avoid unnecessary travels and provide fast and efficient interprofessional and patient/doctor communication [3-6]. Epidemiological data on the rise of cancer incidence and prevalence further enhanced this trend. The American Society of Clinical Oncology (ASCO) predicted the number of cancer survivors would rise from 15.5 million to 20.3 million between 2016 and 2026 [7]. The growing prevalence of cancer survivors combined with the rise in cancer incidence will raise a nearly $50 \%$ increase in cancer care demand which can be challenging to manage for the upcoming possible shortage of oncologists, at least in industrialised countries [8]. Hopefully, telemedicine can help healthcare systems cope with the overwhelming burden of patients numbers [9].

The spread of social media and instant messenger systems provides an excellent opportunity and a helpful tool to healthcare professionals [10]. The Merriam-Webster dictionary defines social media as 'forms of electronic communication through which users create online communities to share information, ideas, personal messages, and other content' [11]. This definition may also apply to instant messenger systems, which are not the same as social media, but they are closely interrelated and somehow fade into each other. Among these tools, WhatsApp instant messaging (WM) system gained a vast diffusion worldwide. WM is a free messaging application, multiplatform, which allows users to send textual messages, images, videos, documents and voice recordings to other users via the Internet, having the telephone number as an identifier [12]. It also allows users to make voice and video calls, send images and documents and is compatible with all smartphones. It differs from 'chat groups', in which the user engages in a more public real-time conversation within a chatroom where everyone on the channel sees everything said by all other users.

The use of a fast-acting, written or video communication system with an app already well known, like WM, is conveniently within reach of a large majority of patients due to its widespread use in peoples' private daily lives. Due to its wide diffusion, there is no need to ask the patient or the caregiver to download or create an account on other Apps, which could be a barrier. These factors have caused a massive boost in web-based educational meetings and the increasing use of telematics communication methods among patients, families, hospitals and different health professionals of the same speciality or belonging to multi-disciplinary teams [10, 13, 14]. However, today an ever-increasing number of apps are offered to oncological health professionals by various providers to provide an efficient patient problem management system $[14,15]$. Scottish researchers reported an analysis of 151 apps targeting people living with and beyond cancer covering various aspects of oncology ranging from disease information and planning medical care to self-monitoring, management strategies and interaction between users and professionals $[3,15]$. Nearly two-thirds of tools have been developed by commercially oriented companies/private individuals, and one-third by non-profit associations, mainly in the USA. Some of the apps are ethically and professionally correct and useful in managing specific clinical settings such as oral cancer therapies, palliative care, exercise and many others [16]. Unfortunately, many apps exploit an engagement system based on the veiled promise, sometimes exaggerated, of empowerment in the struggle against the disease. Furthermore, a minority of apps deplorably report unproven 'cures' for cancer or selling products, such as alkaline waters, to cancer survivors.

In the traditional face-to-face medical visit, the empathic translation of clinical evaluation flowcharts and medical concepts into simple, friendly and non-technical language easily understood by patients and their caregivers represents a cornerstone of the medical art. However, e-medicine communication/information systems often follow a medical logic workflow that is often difficult to interpret by many potential non-medical users and patients for various intuitive reasons. However, the ambitious goal of optimising communication in health care is challenged by the conceptual complexity of many apps, mainly unknown to the general population. For this reason, very often, oncological health professionals have turned to the use of web-based communication and messaging systems already widespread in the world and well known to most of the possible users. Among these tools, the WM instant messenger system has gained enormous popularity due to ease of use, versatility and almost additional costs.

In the last years, the research community analysed the advantages and disadvantages of WM usage in healthcare. Among the negative aspects, scientists included the clinical risks for patients, data security and privacy protection. A working group of the ASCO, the Integrated Media and Technology Committee, reported some suggestions on the responsibly professional use of social media in oncology [17]. While lacking defined guidelines, however, the committee discussed some critical key-issues, including respect for patients' privacy, protection of sensible clinical data in compliance with the legal, regulatory rules and the possible institutional property of communication tools [17]. 
In this narrative review, we report a focus on available data, pros and contras of the unregulated use of WM, in the context of e-medicine, as an interprofessional and doctor/patient communication tool in oncology.

\section{WhatsApp Messenger and business application programming interface (API)}

WM allows health professionals to share data, updates, information, debate health care policy and practice issues [14]. WM also may be used to promote health behaviours, engage with the public and educate and interact with patients, caregivers and students, and colleagues. Although some messaging systems are very similar, if not identical, social media, however potentially carry the additional advantage of being a very efficient management system, is still scientifically unproven in oncology [14, 17]. WM may also be used from any computer station in the WhatsApp Web version that recognises a quick response QR code and connects directly to the user's smartphone. As inferred from the lack of medical literature, not many oncologists know the business version of WM, a free version for smartphones valid for professionals, which permits communication with all standard version WM users [18]. The main difference between regular WM and the Business application is in the profile window. While the standard version contains only name, status and photo, the dedicated business app shows an actual business card, with all the helpful information for being recognised and found by customer such as name, logo, image, description, opening and closing times, website, e-mail address, etc. WM Business API allows appointment management, request medical or laboratory reports, symptoms evaluation, answers to FAQs, statistical analysis, automatic responses and guided workflows, organisation of contacts by categories and broadcast list. In the latter action, the app guarantees individual users' privacy since individual responses are blinded to other users. Healthcare organisations can store massive medical data of patients by integrating WM Business API with other third-party tools.

\section{Health professionals-related issues}

A review by Brazilian investigators reported that WM is a helpful communication tool between health care professionals and patients, caregivers and families, among health care professionals themselves, or as a learning tool for providing information on health care to professionals or the general population [19]. However, the authors felt that high-quality and adequately evaluated research is urgently needed. Patt [5] explored the use of telemedicine and barriers during the COVID-19 pandemic in a statewide, multicentre, high patient volume oncology network. Overall, $96 \%$ of clinicians reported telemedicine, with $33 \%$ using it for more than $25 \%$ of patient visits. Most clinicians said that patients enjoy the benefits of telemedicine, primarily those minimally symptomatic. Its use may cause a decrease in exposure risk and transportation needs. Moreover, it enhanced the presence of caregivers during patients' visits. Availability of technical equipment and Internet resources, such as broadband access, was the most frequent obstacle to patients' access to telemedicine.

\section{Privacy}

WM has an encryption system for both written and video messages to guarantee privacy $[13,18]$. Despite this protection system, several researchers formulated warnings and concerns on the unregulated use of instant messenger systems in health care [1, 19, 20]. Health authorities expressed significant concern concerning confidentiality in WM conversations to avoid putting patients in a vulnerable position and maintain the public's trust in the medical profession [20]. However, the reality is that many doctors regularly use instant messaging systems to communicate data and information and prescriptions to patients even in the face of regulatory agencies' recommendations. A recent Irish study reported that $97 \%$ of doctors surveyed shared sensitive information on instant messenger without the patient's written consent, although 68\% were concerned about this lack of agreement [20]. The use of WM can also carry the risk for health professionals of becoming the target of offenses that may spread on the web [21].

\section{Video-consulting}

The use of WM for patient engagement has begun to appear more and more in medical literature [22]. From a technical point of view, a video consultation is a simple video call. Therefore, different platforms can also be used, including well-known and widespread systems (WhatsApp, Google Hangout, Zoom and many others), or customised and private solutions. For a professional who starts the video consultation activity independently, the best advice is to use the business version WM, which represents a professionally-oriented tool [18]. 


\section{Interprofessional cooperation}

WM may be particularly helpful for interprofessional cooperation. The Italian College of Medical Oncology Directors carried out a descriptive, unplanned investigation to report reactions, attitudes and countermeasures put in place and implemented by 19 medical oncology units facing the COVID-19 outbreak in Southern Italy [23]. All the directors of medical oncology units participate in an informal WM chat. Authors reported 956 WM conversations related to reactions to important events, such as the epidemic spread, Government ordinances and guidelines during 4 weeks of observation. Data showed significant awareness of problems linked to the COVID-19 epidemic among medical oncologists and rapid diffusion of countermeasures. Actions taken were correlated time-wise to important events. Data showed that the number of WM conversations correlated with the volume of activity of oncology units. Textual sentiment analysis of raw data showed a reduction of positive emotions over the weeks. A significant increase in negative emotions was observed as the outbreak impacted the healthcare system. Shaarani et al [24] investigated the prevalence of WM use as an interprofessional communication tool among 5,329 Lebanese physicians explored the dimensions of its use through an e-mail-based questionnaire. Overall, 429 physicians responded completed the survey and $96.5 \%$ of them reported using WM, 72.7\% had WM consultations with colleagues and about 50\% said being members of professional WM groups. Although most participants state to avoid patient identification when sharing information, two-thirds of physicians felt it necessary to develop guidelines, especially for the medico-legal and ethical aspects. The creation of social media-based groups may enhance interprofessional networking and sharing of knowledge among different specialities. Rolls et al [25] published a review of 44 studies present in the medical literature. Overall, the quality of studies was not optimal, and data collection included web-based observation, surveys, interviews, focus groups and diaries. Health care professionals involved in the studies included physicians, nurses, allied health professionals, followed by health care professionals in general, a multi-disciplinary clinical speciality area and midwives. Analysis based on related theories of 'planned behavior' and the 'technology acceptance model' suggests that social media use is dependent on an individual's positive attitude toward and accessibility of the media, which credible peers reinforce. The most frequent driver to create a virtual community is the need for an agorà where health professionals and other users may share and discuss relevant speciality knowledge to enable themselves to make updated practical decisions. Chat participants showed a frequent attitude to read or access speciality-specific clinical information but a low tendency to post data or opinions. Hospitals can leverage the considerable potential of WM to connect with their patients and to organise efficient team coordination. For instance, WM may answer all the necessary information or status of each piece of equipment or coordinate actions. Italian investigators carried out a study aimed to assess the individual and organisational determinants that can trigger or inhibit the use of WM in a hospital setting and which variables managers can exploit to guide professionals' behaviours [26]. A validated questionnaire tested for internal consistency was administered to 191 physicians and nurses in an academic hospital showing that WM is widely used in the hospital due to the subjective perception of its usefulness and numerous practical advantages. Data show that WM may decrease consultation time, number and time spent on phone calls, improve communication and rationalise workflows. The creation of WM groups promotes collaboration, ultimately improving the level of healthcare provided to patients. Moreover, an interplay exists between organisational and individual factors in determining the use of WhatsApp between healthcare professionals and patients. In particular, individual factors play a crucial role as determinants of WM. In contrast, organisational factors play a secondary role, not directly influencing the use of WM but always acting through individual elements. This study also analysed the influence of personal and organisational determinants of WM use in the hospital setting. It provides hospital managers with vital information to manage this phenomenon and implement adequate strategies to increase its potential. An extensive literature review aimed to examine the utilisation of social networks for health professionals' relationships, daily clinical practice, professional networks and education and training to identify areas for future health communication research [13]. Overall, 33 out of 6,977 papers retrieved stated that social networking systems enhanced effective communication and information sharing even if sound scientific evidence lacks. Health professionals used social networking systems to support delivering clinical services, make referrals and share information. They were beneficial to network building and professional collaboration. Social media were considered novel tools to enhance educational interactions among peers, students, instructors and preceptors. The application of social media came with restraints in technical knowledge, concerns on data protection, privacy and liability, issues in professionalism and data protection. Limitations included technical knowledge, professionalism and risks of data protection. The evolving use of such tools necessitates robust research to explore the full potential and the relative effectiveness in professional communication. 


\section{Burnout}

Burnout syndrome and dissatisfaction affect more than 50\% of practicing physicians and oncologists in the USA [27-29]. Only one paper suggests a possible benefit and may reduce the rate of physician burnout even if its use may be linked to pathological addiction [30]. Further studies are needed to clarify a role for instant messengers system in ameliorating burnout.

\section{Research}

Health providers and researchers can use data recorded in WM to find the best treatment options for patients, cooperate with clinical trials or verify patients' journeys [1,10,14,19]. The use of symptom checkers or chatbots to collect and analyse data directly from patients' data on symptoms, family history and risk factors must be scientifically validated.

\section{Teaching}

Clavier et al [31] reported a prospective, randomised multicentre study to measure the impact of a learning programme via WM on clinical reasoning in anaesthesiology residents from four university hospitals in France. Residents were randomised into two groups of online teaching: WM-based one and e-learning control group. The WM group benefited from the daily delivery of teaching documents on the instant messenger system and a weekly clinical case supervised by a senior physician. Residents in the control group access to identical records via a traditional e-learning platform. Medical reasoning was self-assessed online by a script concordance test, and medical knowledge was assessed using multiple-choice questions. The residents also completed an online satisfaction questionnaire. The authors found a difference between the WM and control groups for the script concordance test ( $p=0.006$ ) but no difference for multiple-choice questions. The global satisfaction rate was more significant in the WM than in the control group $(p=0.049)$. Compared to traditional e-learning, the use of WM for teaching residents was associated with worse clinical reasoning despite better global appreciation. The use of WM probably contributes to the dispersion of attention linked to the use of the smartphone. An Indian study evaluated the feasibility and effectiveness of WM in a series of 182 students to provide health education on tobacco and oral cancer as compared to the conventional PowerPoint lessons [32]. Both WM and traditional student groups showed a statistically significant increase in knowledge scores, with substantial improvement in the WM intervention group $(p=0.001)$. However, the validity of WM as a helpful tool in health education strategies has been criticised by other authors [33].

\section{Clinically related issues}

\section{Remote monitoring and consultation}

Any patient can access healthcare services through WM no matter geographical barriers or local time. WM chatbots are also available for mobile consultation to get customised tips or treatment, especially in the current pandemic. On the other hand, doctors can witness a vast number of customers for their services. The widespread use of fast communication tools reduces the gap between low and high-income countries, allowing cancer patients with limited socioeconomic conditions to receive medical assistance closer to patients living in higher conditions [34]. The communication tools, often WM, are helpful in low- and middle-income countries where the incidence and mortality of cancer are high. Yadav and Yadav [35] reported that WM remote monitoring of surgical wounds in patients operated for thyroid cancer was as effective as face-to-face visits with a higher rate of high satisfaction in patients evaluated via web and avoidance of a median of 930 km travel. The authors stressed how these communication tools might be helpful in low- and middle-income countries where the incidence and mortality of cancer are high. An observational cross-sectional study reported positive data for WM to prevent malignant oral cavity disorders in a rural area of India [36]. Overall, 131 patients were screened for oral potentially malignant diseases using photo capturing five regions of the mouths and send via WM to remote examiners and compared to face-to-face clinical evaluation. The kappa reliability score between the diagnoses, based on photo messaging and clinical oral examination, was 0.68 , while sensitivity and specificity were $98 \%$ and $52 \%-64 \%$, respectively. 


\section{Telepathology}

Most of the studies on telepathology have considered results encouraging. However, all concluded that smartphone-assisted telepathology still needs a faster Internet resource and significant improvement in smartphone camera technology to improve the quality and accuracy of the diagnosis. Dixit et al [37] reported the use of WM in telepathology in India. A smartphone camera captured 151 cases of fine-needle aspiration and 10 cases of urine cytology from the ocular of a binocular microscope. Investigators sent images to the cytopathologist at a different location. Results of telepathology were compared to direct microscopic evaluation showing an overall intra-observer concordance of $95.6 \%$ and $90 \%$ for fine-needle aspiration specimens and urine cytology. The authors concluded that further improvement in the smartphone camera resolution and Internet connectivity would enhance the results. Turkish researchers studied 172 specimens to determine intra-observer concordance between traditional microscopic cytopathological diagnoses and diagnoses done on static smartphone images transmitted via WM [38]. This blinded analysis showed an 84\% concordance rate, with a 1.000 Kappa agreement in endoscopic ultrasoundguided fine-needle aspiration specimens and only 0.665 in urine cytology (0.665). The authors concluded that WM is a fast way to transmit images but considered mandatory improvements in smartphone camera technology and transfer software. A Brasilian study evaluated the appropriateness and timeliness of telepathology of 23 endoscopic ultrasound-guided fine-needle aspirations specimens evaluated through WM [39]. An initial diagnosis of malignancy was possible in 14 of 23 patients $(60.8 \%)$. The current study demonstrated the feasibility of a low-cost, Internet-based telepathology system using WM. WM may be helpful to rapidly achieve a second opinion. A total of 247 oral histopathological specimens received a second opinion by 20 different pathologists using WM [40]. Overall, $98 \%$ of 4,795 total second opinions received a concordant diagnosis. The percentage of valid second opinions for malignant tumour specimens was lower than that formulated for cysts of benign or low malignant diseases (75\%-85\% versus $100 \%)$. A positive correlation was observed between correct second opinion and age $(p=0.0143)$ and experience $(p=0.0189)$ of the pathologist. Similar results were reported for gynaecology specimens. Out of 186 gynaecologic pathology cases analysed by conventional microscopy or smartphone camera-derived images and transferred via WM, the smartphone diagnosis was concordant in 179/186 (96.2\%) cases [41]. The intra-observer concordance rate was $97.2 \%$ and $97.6 \%$ for cervical and endometrial myometrial pathology, respectively, and decreased to $80 \%$ for ovarian lesions.

\section{Online surveys, screening and prevention}

WM is a valuable tool to carry out online surveys. An international group of researchers belonging to four scientific societies explored the status of implementing an Enhanced Recovery After Surgery programme in open gynaecologic oncology surgery. The study aimed to provide a worldwide perspective on perioperative practice patterns and decrease the variation in the quality of care [42]. Over 454 respondents representing 62 countries, $37 \%$ reported a programme implementation at their hospitals, even if Western industrialised countries were more compliant (33\%-38\%) than Asian and African ones (10\%-19\%). Data showed a high adherence rate for venous thrombosis prophylaxis, early postsurgical removal of a urinary catheter and precious mobilisations. Poor adherence to the guidelines included bowel preparation, adoption of modern fasting guidelines, carbohydrate loading, use of nasogastric tubes and peritoneal drains, intra-operative temperature monitoring and early feeding. Longitudinal adherence to cancer screening, critical to the success of any faecal test-based screening programme, is often inadequate. Uy et al [43] extensively reviewed 31 out of 2,238 citations concerning cancer screening finding nine papers satisfying inclusion criteria. Five studies examined screening for breast cancer, one for cervical cancer and three for colorectal cancer. Interventions based on sending messages moderately increased adherence to screening programmes which was $0.6 \%-15.0 \%$ higher than for controls. The benefit was observed in various countries, including resource-poor and non-English-speaking populations. Given the lack of data, additional research is needed to quantify this promising intervention's effectiveness better. Lam et al [44] carried out a single-blind, randomised study on reminders for a faecal immunochemical test sent via mobile messengers, such as WM. The study showed a clear improvement of adherence defined as the pick-up and on-time return of tests $(80.3 \%$ versus $59.3 \% ; p<0.001)$, and return rate $(79.9 \%$ versus $57.3 \% ; p<0.001)$ were significantly higher in the WM group compared with the control group. A study carried out at the Johns Hopkins University aimed to evaluate the barriers in research recruitment among racial/ethnic minorities and immigrants [45]. This community-based study examining correlates of cervical cancer screening behaviours showed that online recruitment via WM was an effective recruitment strategy because it built on existing information-sharing norms within the community. A cross-sectional study carried out in Kuwait reached the same conclusions [46]. WM was used to disseminate a survey on oral cancer risk. Among 404 respondents, the prevalence of oral cancer screening was $7.2,7.7 \%$ among nonsmokers, and $5.4 \%$ among smokers. Only $36.6 \%$ were aware of oral cancer, with more nonsmokers (38.9\%) than smokers (29\%). Logistic regression analysis showed that male patients participated more likely to go for screening than females. The likelihood of participation was 
correlated to age, being individuals in the age group of 25-44 years four times more than other age groups $(p<0.012)$. Walter et al [47] studied the effect of a commercially available skin self-monitoring smartphone application among 238 patients with increased risk of melanoma on their decision to seek help for changing skin lesions. Overall, 51 patients (21.4\%) had visits regarding skin changes during the 12 months of follow-up, and 157 individuals (66.0\%) completed one or more questionnaires for follow-up. There were no significant differences in dermatologic consultation rates, size of superficial spreading lesions or psychological harm evaluated by the Melanoma Worry Scale.

\section{Virtual multi-disciplinary tumour board}

An informal WM group was created among 25 specialists, including nine urologists, nine oncologists, three urology residents, three radiotherapists, one general practitioner and a group coordinator [48]. This virtual tumour board discussed clinical cases of genitourinary tumours of particular complexity requiring a multi-disciplinary approach. An evaluation questionnaire was sent after 6 months to evaluate the level of appreciation, reporting an average rating score of appreciation was 7.8 (range: 4-10) according to a 10-point Likert scale. The WM consultation was completed in $90 \%$ of cases, and in $81.8 \%$ of cases, a final agreement on patient management was reached. An average of eight specialists or each case joined the WM-based tumour board, with an average of 17.6 textual interventions for each clinical case. Authors felt WM as a helpful alternative and a rapid complementary communication tool to transfer large amounts of clinical and radiological data.

\section{Patients' related issues}

\section{Patients preference}

Cherrez-Ojeda et al [49] carried out an anonymous cross-sectional survey study in 500 Ecuadorian cancer patients to assess the use of communication technology and patterns of preferences. Only $43 \%$ of participants declared to have web access. WM was the preferred method to receive or ask for information from oncologists in $72 \%$ of cases. Age was correlated to the likelihood of using instant messaging systems. Patients aged between 40 and 64 years were more likely to be interested in receiving information through SMS (odds ratio (OR): 5.09, 95\% $\mathrm{Cl}$ : 1.92-13.32), as well as for asking questions to physicians through this same media (OR: 9.78, 95\% Cl: $3.45-27.67)$ than the oldest group.

\section{Feasibility, useability and acceptability}

Australian investigators reviewed the medical literature concerning the feasibility, useability and acceptability of web technology-based interventions [50, 51]. The review also included data concerning smartphone applications among patients' caregivers. Attrition, recruitment rates and frequency of intervention use were measures of feasibility. Usability was measured by the ease of intervention use and the role of features to minimise errors in use. Acceptability was measured by carers' perception of the appropriateness of the content and their ability to incorporate the intervention into their daily routines. Six out of the 729 articles examined met the inclusion criteria. Feasibility attrition ranged from $14 \%$ to $77 \%$, recruitment rates from $20 \%$ to $66 \%$ and intervention useability varied across studies. Half of the studies implemented measures to improve useability. Overall, carers rated the content of the interventions as appropriate and reported improved knowledge and communication. Data further demonstrated acceptability as carers preferred the flexibility available with web-based interventions. Although current studies have shown the poor quality of evidence, mobile apps may be an efficient solution for caregivers even if dependent on users' needs [52]. Patients found this strategy very convenient and cost-effective and suggested to continue this service in the future, even after the lockdown is lifted [70].

\section{Cancer awareness and risk reduction}

Canadian investigators explored the age differences and degree of confidence with web-based health resources in 371 cancer survivors finding younger age statistically linked to the likelihood of using social media for cancer care [53]. US researchers reviewed 18 studies, including seven randomised trials, using social media among breast cancer women and healthy subjects showing that web-based interventions effectively improve cancer prevention and management [54]. However, the methodology employed to evaluate the impact of social media on cancer-related outcomes needed to be improved in most studies. Authors from the United Arab Emirates reached the same conclusions on the positive role of social media and WM as communication tools to raise awareness and share upcoming breast cancer events and screening [55]. Since many women can discover a breast lump via observation and self palpation, they must be breast cancer aware, i.e., have the 
knowledge, skills and confidence to detect breast changes and present promptly to a healthcare professional [56]. Pereira et al [57] explored the potential of WM as a health education tool to improve women's knowledge on the risk reduction of breast cancer. The authors examined a total of 293 messages dealing with incidence, risk factors, symptoms, myths, protective factors. Although non-specific for the use of WM, many exciting issues emerged from the analysis of the messages, such as social dynamics, uncertainties, spirituality and web or journal news. Communication tools provide the opportunity to exchange personal experiences with disinhibition. However, the presence of a moderator, either a physician or another figure, is necessary to avoid distractions or disengagement, which represent a possible limitation.

\section{Patients' needs}

A descriptive investigation was undertaken at three oncology units to report queries, needs and fears related to the COVID-19 pandemic in patients with cancer and avoid uncontrolled treatment delays or withdrawal, behavioural mistakes and panic [58]. All queries spontaneously delivered through the WM commonly used by patients to communicate with the oncology units were collected and grouped by homology in five categories. Responses to the questions were given according to the Italian Association of Medical Oncology recommendations through WM and subsequent phone calls. Patients were also classified according to the primary tumour site, stage of disease and current treatments. Analysis of the association between these data and queries was carried out. Overall, 446 different patients' WM conversations were analysed between 1 March and 13 March 2020, and comprised the following: requirement of visit delay by patients undergoing oral therapies or in follow-up, delays in chemotherapy or immunotherapy administration, queries about possible immunosuppression and changes in lifestyle or daily activities. Delay requirements were statistically more frequent among patients with prostate or breast cancer than those with lung or pancreatic cancer. Actions taken by oncologists are also reported. The authors concluded that this experience showed WM to be adequate to give a rapid answer to most queries from patients with cancer in the COVID-19 pandemic scenario. COVID-19 pandemic caused several countermeasures such as strict restriction or complete limitation of hospital visits to relatives. WM was employed to allow family members to participate in clinical rounds in an acute palliative care unit and hospice [59]. Although most of the interviewed family members reported a positive impression, the actual presence bedside was considered irreplaceable. De Camargos et al [60] assessed happiness/satisfaction with life and positive and negative effects in 342 cancer patients and 126 informal caregivers compared with healthy people employing WM. In this cross-sectional study, patients and informal caregivers reported at multivariate analysis more positive emotional states than the healthy people despite lower positive affect scores and higher negative affect scores. Although cancer patients experience distressing symptoms and health-related changes in their quality of life, they may report positive emotional states. UK investigators carried out an in-depth qualitative study to explore how women use and experience social media and instant messenger systems, including WM, to self-manage their psychosocial needs and support self-management across the breast cancer continuum [61]. Results showed that such media allowed women living with or after breast cancer to timely self-manage some needs and receive support. However, women experienced social media as empowering and dislocating, as their everyday cancer experiences impacted their engagement. The authors concluded that professionals involved in the cancer health care continuum should improve their awareness of using such tools to initiate value-free discussions and create the space necessary for a tailored and timely self-managed approach to their unique psychosocial needs for women. Recently, the World Health Organization (WHO) created a tool to provide updates and verified data to WhatsApp users, being able to reach more people at once in a simple and effective way. At first glance it looks like a contact like many others saved in the address book but to respond to messages sent by users there is a software ready to provide updates screened directly from the WHO [62].

\section{Clinical monitoring}

A literature review of 26 articles addressed the use of mobile applications to monitor adherence and support in the self-management of complications associated with chemotherapy treatments [63]. The authors identified 16 different mobile applications, which resulted in an effective monitoring process. These apps should be tested in a real-world setting since the evaluation of efficacy in a scientific programme may represent a possible limitation and does not necessarily mean effectiveness in routine practice. Another systematic review by Brasilian investigators reported the usefulness of mobile apps to spread knowledge for breast cancer patients and symptoms reporting management of treatment-related side effects and self-care promotion [64]. Similar conclusions were reported of breast cancer patients in United Arab Emirates [55]. An Indonesia scoping review analysed the use of mobile apps in the management of home palliative care, reporting they can improve the delivery of care, primarily through the improvement of knowledge among family carers [65]. These statements have been 
confirmed by a Canadian descriptive, exploratory, proof-of-concept study carried out to evaluate the efficacy of telemedicine in guiding familiar caregivers or nurses in delivering palliate care at distant homes [66]. Patients and caregivers reported a positive effect and convenience of such intervention. The main aspects reported through questionnaires and interviews included communication, logistics, technical issues, trust and insecurity. Among social media, WM was successfully employed in the remote evaluation of haematuria in a series of 212 patients reducing unnecessary costs of services in cases with mild clinical significance [67].

\section{Pitfalls}

Patients with severe or potentially severe clinical picture are not suitable for telemedicine or other communication tool evaluation, including WM, since they most often require a physical examination, protocol-driven procedures and decisive in-hospital interventions. Clinical emergencies, cognitive alterations, comprehension barriers, lack of access to virtual visit technology represent medical situations that necessarily require a face-to-face visit to guarantee adequate assistance. A possible exception is represented by the management at the home of patients in palliative therapy or in a terminal state that can be managed effectively via the web [68, 69]. Australian researchers showed that implementing a palliative care website requires considerable networking skills, resources, commitment and flexibility to adapt to different user groups such as practitioners, other professional figures, allied health professional groups, patients and their caregivers [69].

\section{Conclusions}

Instant messaging systems in cancer care facilitate improved communication and support between patients, caregivers and clinicians, and consequently a better patient care.

These benefits are particularly useful in rural areas and low- and middle-income countries to partially overcome economic and distance barriers to care, although in these countries the availability of the Internet and the costs associated with connectivity and devices may be a limitation.

In addition, in the COVID-19 pandemic scenario, the rapid transition to electronic visits for non-treatment cancer patients provided an immediate benefit by reducing the unnecessary risk of exposure to infection, overcoming the transport barriers faced by patients and caregivers. A deeper understanding of the impact of e-medicine on clinical outcomes, quality of care and access to care is mandatory to delineate the role of telemedicine and communication tools in the post-pandemic era. Clinicians need to carefully manage instant messaging systems to improve patient care and clinical outcomes. However, the unregulated use of WM, the most cited communication tool in healthcare, raises legal, regulatory and ethical concerns. Consequently, there is a huge need for general guidelines, but this is a difficult goal to achieve. In conclusion, although a significant number of articles have studied the role of social networks in connecting patients to health professionals, there is still a lack of scientific information and data on their uses, benefits and limitations in connecting health professionals for professional communication only. The role of instant messaging systems in cancer practice and research needs to be clarified.

\section{Authors' contributions}

Conceptualisation: VG and DP; methodology: DP and AF; formal analysis: AF, VG and MRV; writing - original draft preparation: VG and DP; writing - review and editing: all authors have read and agreed to the published version of the manuscript.

\section{Funding}

This research received no external funding. 


\section{Institutional review board statement}

Not applicable.

\section{Conflicts of interest}

None of the authors have any personal conflicts of interest to declare.

\section{References}

1. Sedrak MS, Attai DJ, and George K, et al (2018) Integrating social media in modern oncology practice and research Am Soc Clin Oncol Edu Book Am So Clin Oncol Annu Meet 38 894-902 https://doi.org/10.1200/EDBK_204453

2. Riera R, Bagattini ÂM, and Pacheco RL, et al (2021) Delays and disruptions in cancer health care due to COVID-19 pandemic: systematic review JCO Glob Oncol 7 311-323 https://doi.org/10.1200/G0.20.00639 PMID: 33617304 PMCID: 8081532

3. Adam R, McMichael D, and Powell D, et al (2019) Publicly available apps for cancer survivors: a scoping review BMJ Open 9 e032510 https://doi.org/10.1136/bmjopen-2019-032510 PMID: 31575584 PMCID: 6773350

4. Healthcare Finance News (2019) Telemedicine Is Poised to Grow as Its Popularity Increases among Physicians and Patients https://www. healthcarefinancenews.com/news/telemedicine-poised-grow-its-popularity-increases-among-physicians-and-patients Date accessed: $7 / 05 / 21$

5. Patt D (2020) Using clinical informatics to navigate a crisis: how technology and policy change can influence cancer care delivery JCO Clin Cancer Inform 4 318-320 https://doi.org/10.1200/CCI.20.00033 PMID: 32282231 PMCID: 7259878

6. Other Mobile Applications https://www.cancer.net/navigating-cancer-care/managing-your-care/other-mobile-applications Date accessed: 7/05/21

7. Miller KD, Nogueira L, and Mariotto AB, et al (2019) Cancer treatment and survivorship statistics, 2019 CA Cancer J Clin $69363-385$ https://doi.org/10.3322/caac.21565 PMID: 31184787

8. AHA News AAMC Updates Physician Shortage Projections https://www.aha.org/news/headline/2019-04-25-aamc-updates-physicianshortage-projections Date accessed: 7/05/21

9. McCall B (2020) Could telemedicine solve the cancer backlog? Lancet Digit Health 2 e456-e457 https://doi.org/10.1016/S25897500(20)30194-1

10. Knudsen KE, Willman C, and Winn R (2021) Optimizing the use of telemedicine in oncology care: postpandemic opportunities Clin Cancer Res Off J Am Assoc Cancer Res 27 933-936 https://doi.org/10.1158/1078-0432.CCR-20-3758

11. Definition of Social Media https://www.merriam-webster.com/dictionary/social+media Date accessed: 7/05/21

12. WhatsApp https://www.whatsapp.com/?lang=en Date accessed: 7/05/21

13. Chan WS and Leung AY (2018) Use of social network sites for communication among health professionals: systematic review J Med Internet Res 20 e117 https://doi.org/10.2196/jmir.8382 PMID: 29592845 PMCID: 5895921

14. Masoni M and Guelfi MR (2020) WhatsApp and other messaging apps in medicine: opportunities and risks Intern Emerg Med $15171-173$ https://doi.org/10.1007/s11739-020-02292-5 PMID: 32062746

15. Apps to Support Cancer Patients https://www.mkuh.nhs.uk/ Date accessed: 7/05/21 
16. Skrabal Ross X, Gunn KM, and Patterson P, et al (2018) Mobile-based oral chemotherapy adherence-enhancing interventions: scoping review JMIR MHealth UHealth 6 e11724 https://doi.org/10.2196/11724 PMID: 30578182 PMCID: 6320412

17. Dizon DS, Graham D, and Thompson MA, et al (2012) Practical guidance: the use of social media in oncology practice J Oncol Pract 8 e114-e124 https://doi.org/10.1200/JOP.2012.000610 PMCID: 3439237

18. WhatsApp Business https://www.whatsapp.com/business/?lang=en Date accessed: 7/05/21

19. Giordano V, Koch H, and Godoy-Santos A, et al (2017) WhatsApp messenger as an adjunctive tool for telemedicine: an overview Interact J Med Res 6, e11 https://doi.org/10.2196/ijmr.6214 PMID: 28733273 PMCID: 5544893

20. O'Sullivan DM, O'Sullivan E, and O'Connor M, et al (2017) WhatsApp Doc? BMJ Innov 3 238-239 https://doi.org/10.1136/bmjinnov-2017-000239

21. The Times Ten Doctors 'in Offensive WhatsApp Group' https://www.thetimes.co.uk/article/ten-doctors-in-offensive-whatsapp-groupObzct3pxf Date accessed: 7/05/21

22. Maudgal S (2018) Patient engagement for metastatic breast cancer patients: WhatsApp counseling J Glob Oncol 4 114s https://doi. org/10.1200/jgo.18.13700

23. Blasi L, Bordonaro R, and Borsellino N, et al (2020) Reactions and countermeasures of medical oncologists towards the incoming COVID-19 pandemic: a WhatsApp messenger-based report from the Italian college of chief medical oncologists Ecancermedicalscience 141046 https://doi.org/10.3332/ecancer.2020.1046 PMID: 32565899 PMCID: 7289620

24. Shaarani I, El-Kantar A, and Hamzeh N, et al (2020) Interprofessional communication of physicians using WhatsApp: physicians' perspective Telemed J E-Health Off J Am Telemed Assoc 26 1257-1264 https://doi.org/10.1089/tmj.2019.0216

25. Rolls K, Hansen M, and Jackson D, et al (2016) How health care professionals use social media to create virtual communities: an integrative review J Med Internet Res 18 e166 https://doi.org/10.2196/jmir.5312 PMID: 27328967 PMCID: 4933801

26. De Benedictis A, Lettieri E, and Masella C, et al (2019) WhatsApp in hospital? An empirical investigation of individual and organizational determinants to use PLoS One 14 e0209873 https://doi.org/10.1371/journal.pone.0209873 PMID: 30633754 PMCID: 6329505

27. Whippen DA and Canellos GP (1991) Burnout syndrome in the practice of oncology: results of a random survey of 1,000 oncologists J Clin Onco 9 1916-1920 https://doi.org/10.1200/JCO.1991.9.10.1916

28. Shanafelt TD, Dyrbye LN, and West CP (2017) Addressing physician burnout: the way forward JAMA 317901 https://doi.org/10.1001/ jama.2017.0076 PMID: 28196201

29. Shanafelt TD, Hasan O, and Dyrbye LN, et al (2015) Changes in burnout and satisfaction with work-life balance in physicians and the general US working population between 2011 and 2014 Mayo Clin Proc 90 1600-1613 https://doi.org/10.1016/j.mayocp.2015.08.023 PMID: 26653297

30. Han R, Xu J, and Ge Y, et al (2020) The impact of social media use on job burnout: the role of social comparison Front Public Health 8 588097 https://doi.org/10.3389/fpubh.2020.588097 PMID: 33330332 PMCID: 7710858

31. Clavier T, Ramen J, and Dureuil B, et al (2019) Use of the smartphone app WhatsApp as an e-learning method for medical residents: multicenter controlled randomized trial JMIR MHealth UHealth 7 e12825 https://doi.org/10.2196/12825 PMID: 30964435 PMCID: 6477573

32. Nayak PP, Nayak SS, and Sathiyabalan D, et al (2018) Assessing the feasibility and effectiveness of an app in improving knowledge on oral cancer-an interventional study J Cancer Educ 33 1250-1254 https://doi.org/10.1007/s13187-017-1239-y

33. Bridge P, Carmichael MA, and Callender J, et al (2018) Internationalising research methods teaching of undergraduate health professionals J Med Imaging Radiat Sci 49 97-105 https://doi.org/10.1016/j.jmir.2017.11.003 PMID: 30479296 
34. Williams V and Kovarik C (2018) WhatsApp: an innovative tool for dermatology care in limited resource settings Telemed J E-Health Off J Am Telemed Assoc 24 464-468 https://doi.org/10.1089/tmj.2017.0197

35. Yadav SK and Yadav N (2021) Continuity of cancer care in the era of COVID-19 pandemic: role of social media in low- and middleincome countries World J Clin Cases 9 291-295 https://doi.org/10.12998/wjcc.v9.i2.291 PMID: 33521097 PMCID: 7812886

36. Vinayagamoorthy K, Acharya S, and Kumar M, et al (2019) Efficacy of a remote screening model for oral potentially malignant disorders using a free messaging application: a diagnostic test for accuracy study Aust J Rural Health 27 170-176 https://doi.org/10.1111/ ajr.12496 PMID: 30942518

37. Dixit S, Tanveer N, and Kumar H, et al (2020) Smartphone-assisted telecytopathology: an intraobserver concordance study Acta Cytol 64 399-405 https://doi.org/10.1159/000506145 PMID: 32074596

38. Sahin D, Hacisalihoglu UP, and Kirimlioglu, SH (2018) Telecytology: is it possible with smartphone images? Diagn Cytopathol 46 40-46 https://doi.org/10.1002/dc.23851

39. Machado RS, Richa R, and Callegari F, et al (2019) Instant messenger smartphone application for endosonographer/cytopathologist real-time interaction at a distance in EUS-FNA for solid pancreatic lesions Endosc Int Open 7 E1027-E1030 https://doi. org/10.1055/a-0965-6631 PMID: 31404419 PMCID: 6687484

40. Sarode SC, Sarode GS, and Anand R, et al (2017) WhatsApp is an effective tool for obtaining second opinion in oral pathology practice J Oral Pathol Med 46 513-519 https://doi.org/10.1111/jop.12515

41. Kumar H, Tanveer N, and Dixit S, et al (2020) Smartphone-assisted tele-gynepathology: a pilot study J Obstet Gynaecol Res 46 1879-1884 https://doi.org/10.1111/jog.14347 PMID: 32875651

42. Bhandoria GP, Bhandarkar $P$, and Ahuja V, et al (2020) Enhanced recovery after surgery (ERAS) in gynecologic oncology: an international survey of peri-operative practice Int J Gynecol Cancer 30 1471-1478 https://doi.org/10.1136/ijgc-2020-001683 PMID: 32753562

43. Uy C, Lopez J, and Trinh-Shevrin C, et al (2017) Text messaging interventions on cancer screening rates: a systematic review J Med Internet Res 19 e296 https://doi.org/10.2196/jmir.7893 PMID: 28838885 PMCID: 5590008

44. Lam TYT, Wu PI, and Tang RSY, et al (2021) Mobile messenger-initiated reminders improve longitudinal adherence in a communitybased, opportunistic colorectal cancer screening program: a single-blind, crossover randomized controlled study Cancer 127 914-921 https://doi.org/10.1002/cncr.33336

45. Cudjoe J, Turkson-Ocran RA, and Ezeigwe AK, et al (2019) Recruiting African immigrant women for community-based cancer prevention studies: lessons learned from the AfroPap study J Community Health 44 1019-1026 https://doi.org/10.1007/s10900-01900677-y PMID: 31102117 PMCID: 6708449

46. Alkhubaizi Q, Khalaf ME, and Dashti H, et al (2018) Oral cancer screening among smokers and nonsmokers J Int Soc Prev Community Dent 8 553-559 https://doi.org/10.4103/jispcd.JISPCD_197_18 PMCID: 6280576

47. Walter FM, Pannebakker MM, and Barclay ME, et al (2020) Effect of a skin self-monitoring smartphone application on time to physician consultation among patients with possible melanoma: a phase 2 randomized clinical trial JAMA Netw Open 3 e200001 https://doi. org/10.1001/jamanetworkopen.2020.0001 PMID: 32101302 PMCID: 7137684

48. Di Maida F, Scalici Gesolfo C, and Fazio I, et al (2017) Whatsapp messenger as a tool for the multidisciplinary management in everyday clinical practice Eur Urol Suppl 16 e1445-e1446 https://doi.org/10.1016/S1569-9056(17)30881-3

49. Cherrez Ojeda I, Vanegas E, and Torres M, et al (2018) Ecuadorian cancer patients' preference for information and communication technologies: cross-sectional study J Med Internet Res 20 e50 https://doi.org/10.2196/jmir.8485 
50. Heynsbergh N, Heckel L, and Botti M, et al (2018) Feasibility, useability and acceptability of technology-based interventions for informal cancer carers: a systematic review BMC Cancer 18244 https://doi.org/10.1186/s12885-018-4160-9 PMID: 29499663 PMCID: 5834845

51. Heynsbergh N, Heckel L, and Botti M, et al (2019) A smartphone app to support carers of people living with cancer: a feasibility and usability study JMIR Cancer 5 e11779 https://doi.org/10.2196/11779 PMID: 30702432 PMCID: 6374733

52. Sala-González M, Pérez-Jover $V$, and Guilabert M, et al (2021) Mobile apps for helping informal caregivers: a systematic review Int J Environ Res Public Health 18 https://doi.org/10.3390/ijerph18041702 PMID: 33578819 PMCID: 7916631

53. Eng L, Bender J, and Hueniken K, et al (2020) Age differences in patterns and confidence of using Internet and social media for cancercare among cancer survivors J Geriatr Oncol 11 1011-1019 https://doi.org/10.1016/j.jgo.2020.02.011 PMID: 32169545

54. Han CJ, Lee YJ, and Demiris G (2018) Interventions using social media for cancer prevention and management: a systematic review Cancer Nurs 41 E19-E31 https://doi.org/10.1097/NCC.0000000000000534 PMCID: 5787052

55. Abu Awwad D, Hossain SZ, and Mackey M, et al (2020) Women's breast cancer knowledge and health communication in the United Arab Emirates Healthcare (Basel) 8 https://doi.org/10.3390/healthcare8040495

56. O'Mahony M, Comber H, and Fitzgerald T, et al (2017) Interventions for raising breast cancer awareness in women Cochrane Database Syst Rev 2 CD011396 https://doi.org/10.1002/14651858.CD011396.pub2

57. Pereira AAC, Destro JR, and Picinin Bernuci M, et al (2020) Effects of a WhatsApp-delivered education intervention to enhance breast cancer knowledge in women: mixed-methods study JMIR MHealth UHealth 8 e17430 https://doi.org/10.2196/17430 PMID: 32706726 PMCID: 7404019

58. Gebbia V, Piazza D, and Valerio MR, et al (2020) Patients with cancer and COVID-19: a WhatsApp Messenger-based survey of patients' queries, needs, fears, and actions taken JCO Glob Oncol 6 722-729 https://doi.org/10.1200/GO.20.00118 PMID: 32412811 PMCID: 7271316

59. Mercadante S, Adile C, and Ferrera P, et al (2020) Palliative care in the time of COVID-19 J Pain Symptom Manage 60 e79-e80 https:// doi.org/10.1016/j.jpainsymman.2020.04.025 PMID: 32376263 PMCID: 7196549

60. de Camargos MG, Paiva BSR, and de Oliveira MA, et al (2020) An explorative analysis of the differences in levels of happiness between cancer patients, informal caregivers and the general population BMC Palliat Care 19106 https://doi.org/10.1186/s12904-020-00594-1 PMID: 32652992 PMCID: 7354680

61. Ure C, Cooper-Ryan AM and Condie J, et al (2020) Exploring strategies for using social media to self-manage health care when living with and beyond breast cancer: in-depth qualitative study J Med Internet Res 22 e16902 https://doi.org/10.2196/16902 PMID: 32364510 PMCID: $\underline{7281122}$

62. WHO health alert brings COVID-19 facts to billions via WhatsApp https://www.who.int/news-room/feature-stories/detail/whohealth-alert-brings-covid-19-facts-to-billions-via-whatsapp Date accessed: 7/5/21

63. Magalhães B, Fernandes C, and Martinez-Galiano JM, et al (2020) Exploring the use of mobile applications by cancer patients undergoing chemotherapy: a scoping review Int J Med Inf 144104293 https://doi.org/10.1016/j.ijmedinf.2020.104293

64. Cruz FOAM, Vilela RA, and Ferreira EB, et al (2019) Evidence on the use of mobile apps during the treatment of breast cancer: systematic review JMIR MHealth UHealth 7 e13245 https://doi.org/10.2196/13245 PMID: 31456578 PMCID: 6734853

65. Putranto, D and Rochmawati, E (2020) Mobile applications for managing symptoms of patients with cancer at home: a scoping review Int J Nurs Pract 26 e12842 https://doi.org/10.1111/ijn.12842 PMID: 32347599

66. Read Paul L, Salmon C, and Sinnarajah A, et al (2019) Web-based videoconferencing for rural palliative care consultation with elderly patients at home Support Care Cancer 27 3321-3330 https://doi.org/10.1007/s00520-018-4580-8 PMID: 30613908 
67. Sener TE, Butticè S, and Sahin B, et al (2018) WhatsApp use in the evaluation of hematuria. Int J Med Inf 111 17-23 https://doi. org/10.1016/j.jijmedinf.2017.12.011

68. Lai L, Sato R, and He S, et al (2020) Usage patterns of a web-based palliative care content platform (PalliCOVID) during the COVID-19 pandemic J Pain Symptom Manage 60 e20-e27 https://doi.org/10.1016/j.jpainsymman.2020.07.016 PMID: 32730951 PMCID: 7383177

69. Street AF, Swift K, and Annells M, et al (2007) Developing a web-based information resource for palliative care: an action-research inspired approach BMC Med Inform Decis Mak 726 https://doi.org/10.1186/1472-6947-7-26 PMID: 17854509 PMCID: 2194759

70. Sharma Poudyal B, Gyawali B and Rondelli D (2020) Rapidly established telehealth care for blood cancer patients in Nepal during the COVID-19 pandemic using the free app Viber ecancer 14 ed104 https://doi.org/10.3332/ecancer.2020.ed104 